> Comment préserver l'accessibilité aux nouveaux médicaments pour tous les patients qui en ont besoin? La question se pose à la lumière des prix exorbitants auxquels les thérapies géniques, mais aussi les biothérapies et de nouvelles molécules chimiques, sont commercialisées. L'analyse montre qu'à court/moyen terme, l'arrivée sur le marché d'un nombre croissant de nouveaux traitements à haute valeur ajoutée mais à des prix très élevés deviendra insoutenable pour les organismes payeurs qui sous-tendent notre système de santé. Les arguments de rentabilité invoqués par les sociétés pharmaceutiques pour justifier les prix proposés sont à tout le moins contestables, d'autant plus qu'ils ne sont pas documentés de façon transparente et que cette industrie jouit déjà de marges bénéficiaires considérables, en comparaison de celles d'autres secteurs du monde économique. Quant aux promesses de guérison définitive assurée par certains traitements, il faudra encore attendre quelques années pour en vérifier le fondement. À l'aube d'une réforme qui apparaît incontournable, nous formulons plusieurs propositions pour faire avancer le débat, en particulier : (1) mettre en œuvre la résolution avancée par plusieurs états membres de l'Organisation mondiale de la santé (OMS) pour assurer la transparence des coûts de développement des nouveaux médicaments; (2) imposer une clause de prix raisonnable dans les accords qui régissent le transfert de technologies entre les institutions académiques soutenues par des fonds publics et les entreprises privées; (3) instituer une instance européenne commune de négociation des prix des médicaments adaptée aux conditions socioéconomiques de chaque pays; (4) conditionner le remboursement des traitements innovants à leur commercialisation par des entreprises - ou des filiales de celles-ci - répondant à des normes certifiant leur responsabilité sociétale et (5) développer en Europe des partenariats public-privé à but non lucratif, pour la création de quelques unités de production de biothérapies et thérapies géniques. L'objectif général de ces mesures serait de fixer un cadre qui permette de définir des prix qui garantissent un juste retour de l'investissement public vers le système de santé tout en assurant pour les entreprises un profit d'un niveau conforme au marché et une incitation suffisante à investir dans les domaines prioritaires pour la santé publique. <

Vignette (Photo @ Inserm - Patrice Latron).
${ }^{1}$ Collège de France, Assistance Publique des Hôpitaux de Paris, Hôpital Necker-Enfants Malades,

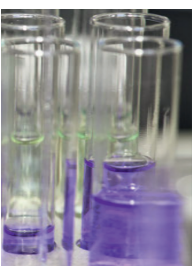
Unité d'Immunologie-Hématologie Pédiatrique, Imagine Institute, Inserm UMR 1163, 75015 Paris, France.

${ }^{2} \varepsilon$ cares, Solvay Brussels School of Economics and Management, Université libre de Bruxelles, Belgique.

${ }^{3}$ I3h Institute, Université libre de Bruxelles, Belgique. alain.fischer@aphp.fr mathias.dewatripont@ulb.be mgoldman@i3health.eu

Les avancées de la recherche biomédicale ont conduit à la mise sur le marché d'un nombre croissant de nouveaux médicaments à haute valeur ajoutée. Les exemples abondent: il suffit de mentionner le traitement curatif de l'hépatite C, l'immunothérapie des cancers, des maladies auto-immunes et inflammatoires, et les thérapies ciblées des maladies rares. Ces innovations concernent les classes de médicaments classiques, mais aussi les biothérapies et désormais les thérapies géniques [1-3]. Les perspectives sont particulièrement encourageantes si l'on se fonde sur le nombre d'essais cliniques en cours, notamment dans le domaine du cancer et des maladies rares. Ainsi, il est estimé que 30 à 60 produits de thérapie génique destinés à ces indications devraient atteindre le marché dans les 10 années à venir et concerner plusieurs centaines de milliers de patients à travers le monde [4]. Voilà qui explique les investissements considérables de l'industrie pharmaceutique dans ces thérapeutiques innovantes, en particulier à travers le rachat de firmes de biotechnologie; ainsi, une grande compagnie pharmaceutique a consacré 8,9 milliards de dollars américains (\$), soit environ 8,2 milliards d'euros $(€)$ à l'acquisition de la société de biotechnologie qui a développé le produit de thérapie génique Zolgensma ${ }^{\circledR}$ pour l'amyotrophie spinale (SMA) $[29,30](\rightarrow)$.

$(\rightarrow)$ Voir les Synthèses de M. Barkats et S. Braun, $\mathrm{m} / \mathrm{s} \mathrm{n}^{\circ} 2$, février 2020 , pages 137 et 141

Parallèlement, les prix des traitements de ce type mis récemment sur le marché atteignent des montants sans 
précédent, que ce soit dans le domaine de la thérapie génique ou pour des molécules chimiques innovantes [5,6] (Tableau I). Dernier exemple particulièrement éloquent: le nouveau médicament contre la mucoviscidose, Trikafta $^{\circledR}$, qui corrige et potentialise l'action de la protéine CFTR (cystic fibrosis transmembrane conductance regulator), anormale chez les patients, est commercialisé au prix d'environ $300000 \$$ (un peu plus de $275000 €$ ) par patient et par an aux ÉtatsUnis $[7,8]$ ! Les conséquences de cette situation sont multiples et préoccupantes : cercle vicieux d'augmentation des prix fondée sur le bénéfice invoqué d'un nouveau médicament comparé au précédent, déjà onéreux. Cet « effet d'ancrage » [9] est illustré par le traitement de la SMA par thérapie génique (Zolgensma ${ }^{\circledR}, 2,1$ millions \$/an ou un peu plus de 1,8 million $€ / a n)$, «justifié » par le coût d'un traitement alternatif par Spinraza ${ }^{\circledR}$ - une approche consistant en l'injection d'oligonucléotides - au prix de 250000 \$/an (environ $230000 € /$ an). De manière générale, le prix des thérapies géniques des maladies rares n'a fait que croître au fur et à mesure de la commercialisation de chaque produit : de 650000 \$ (pour le déficit immunitaire en adénosine déaminase $[A D A]$ ) à $2100000 \$$. Rien ne garantit que cette inflation se ralentira à mesure que les marchés s'élargiront.

Ces prix exorbitants conduisent immanquablement et paradoxalement à une restriction d'utilisation, ne serait-ce qu'en raison d'un retard à la mise sur le marché lié aux négociations ardues entre les organismes payeurs et les compagnies pharmaceutiques. Le désespoir des parents d'enfants privés de traitements supposés salvateurs conduit à des situations encore inimaginables il y a quelques années; on songe ici au financement participatif de traitements non-remboursés par les autorités de santé ou, pire encore, au tirage au sort d'enfants, heureux élus d'un traitement « offert» par l'entreprise productrice, comme c'est le cas du traitement de l'amyotrophie spinale par Zolgensma ${ }^{\circledR}[30](\rightarrow)$.

\section{Comment en est-on arrivé là ?}

$(\rightarrow)$ Voir l'Encadré de la page 143 dans la Synthèse de $S$. Braun, $m / s n^{\circ} 2$, février 2020 , pages 141-6

Les arguments avancés par les industriels pour justifier les prix astronomiques des traitements les plus innovants sont connus: caractère «transformant» de la vie des patients, coût de la recherche qui les sous-tend, coût de production de médicaments de plus en plus sophistiqués, étroitesse du marché, nécessité de promouvoir et récompenser l'innovation.

La recherche est évidemment coûteuse et nécessite des investissements considérables et risqués, essentiellement dans les phases d'expérimentation clinique préalables à l'approbation par les autorités réglementaires. Toutefois, nous ne disposons pas de données chiffrées précises qui permettraient d'évaluer les répercussions de ces investissements sur les prix des médicaments. II faut noter que les frais de recherche et de développement ne représentent qu'environ $20 \%$ du prix de vente [10], soit bien moins que les coûts de marketing et de vente. De plus, la contribution académique au développement des médicaments nouveaux les plus récents est considérable [11-13]. L'OMS a récemment plaidé pour plus de transparence dans ce domaine, mais il reste à voir comment sa recommandation sera mise en pratique [14]. Aussi, les contributions des organisations publiques et philanthropiques qui financent la recherche académique à l'origine de la majorité des découvertes sont fréquemment passées sous silence dans le raisonnement justifiant les coûts. Rappelons pourtant ici que la Commission Européenne a financé à hauteur de plus de 1 milliard d'euros la recherche en thérapie génique de 2007 à 2017 (base de données Cordis). L'argent des citoyens ainsi investi dans la recherche ne devrait-il pas donner lieu impérativement à un retour vers la société lorsque cette recherche aboutit à de nouveaux traitements?

Qu'en est-il de l'argument de la taille réduite des marchés concernés? S'il est vrai que les premières thérapies géniques approuvées s'appliquaient à des maladies rares, voire ultra-rares, les nouvelles indications comme la $\beta$-thalassémie et bientôt les hémophilies concernent des populations beaucoup plus nombreuses, sans parler des patients atteints d'hémopathies lymphoïdes malignes désormais traités par les cellules CAR (chimeric antigen receptor) T [4], II s'avère d'ailleurs que la relation entre le prix affiché des traitements et le nombre de patients éligibles est loin d'être évidente tant pour les thérapies géniques (Tableau l) que pour certaines thérapies fondées sur l'utilisation d'anticorps monoclonaux pour traiter par exemple la maladie de Crohn ou l'hémoglobinurie paroxystique nocturne, ou encore la nouvelle trithérapie désormais utilisée pour traiter la mucoviscidose (Tableau II) [15]. Quid de l'argument, souvent présenté comme définitif : la valeur de ces traitements pour les patients [16] ? II est vrai que les thérapies géniques sont désormais potentiellement curatives, un seul traitement pouvant suffire à sauver la vie du patient ou à sauvegarder une fonction essentielle comme la vision. Relevons toutefois que l'assurance d'une guérison définitive n'est pas toujours établie, le recul des traitements de patients n'excédant que rarement 5 ans. Il existe des modèles permettant de quantifier la valeur d'un nouveau médicament et d'en déduire un prix (value-based pricing), mais on oublie souvent dans le raisonnement un élément essentiel, souligné par l'Institute for clinical and economic review (ICER) aux États-Unis : le prix calculé d'un médicament sur la base de son efficacité thérapeutique est en fait le prix maximum que peut revendiquer l'industriel et non le prix imposable aux organismes payeurs. On peut aussi faire remarquer que, fort heureusement, la capacité curative d'un traitement médicamenteux n'est pas toujours associée à un prix élevé. Il en est ainsi de l'antibiothérapie, qui, bien que sauvant la vie d'un patient atteint de septicémie, a un coût raisonnable, ou de l'injection de noradrénaline qui sauve la vie d'un patient en état de choc anaphylactique. 


\begin{tabular}{|c|c|c|c|c|}
\hline Maladie & Produit & $\begin{array}{l}\text { Année de } \\
\text { commercialisation }\end{array}$ & $\begin{array}{l}\text { Prix affiché } \\
\text { ( } € \text { et US \$) }\end{array}$ & $\begin{array}{c}\text { Nombre estimé de patients éligibles } \\
\text { (Amérique du Nord + Europe) }\end{array}$ \\
\hline $\begin{array}{l}\text { Déficit immunitaire combiné } \\
\text { sévère (SCID), ADA }\end{array}$ & Strimvelis ${ }^{\circledR}$ & 2016 (Eur) & $594000 €$ & $30-40$ \\
\hline Amaurose de Leber & Luxturna ${ }^{\circledR}$ & $\begin{array}{l}2017 \text { (USA) } \\
2018 \text { (Eur) }\end{array}$ & $850000 \$$ & $>2000$ \\
\hline $\begin{array}{l}\text { Leucémie aiguë } \\
\text { lymphoblastique } B *\end{array}$ & Kymriah ${ }^{\circledR}$ & $\begin{array}{l}2017 \text { (USA) } \\
2018 \text { (Eur) }\end{array}$ & $470000 \$$ & 1000 \\
\hline $\begin{array}{l}\text { Lymphome B à } \\
\text { grandes cellules** }\end{array}$ & $\begin{array}{l}\text { Kymriah }{ }^{\circledR} \\
\text { Yescarta }^{\circledR}\end{array}$ & $\begin{array}{l}2017 \text { (USA) } \\
2018 \text { (Eur) } \\
2017 \text { (USA) } \\
2018 \text { (Eur) }\end{array}$ & $\begin{array}{l}373000 \$ \\
375000 \$\end{array}$ & 1200 \\
\hline Amyotrophie spinale & Zolgensma ${ }^{\circledR}$ & 2019 (USA) & $2,1 \mathrm{Md} \$$ & $>1500$ \\
\hline$\beta$ thalassémie ${ }^{\star \star \star}$ & Zynteglo ${ }^{\circledR}$ & 2019 (Eur) & $1,5 \mathrm{~m} €$ & $>10000$ \\
\hline
\end{tabular}

Tableau I. Prix affiché des thérapies géniques. * : patients âgés de plus de 25 ans après allogreffe de cellules souches hématopoïétiques ou après seconde rechute; ${ }^{* *}$ : en $3^{e}$ ligne de traitement, maladie réfractaire ou en rechute; ${ }^{* \star}: \beta$ thalassémie non $\mathrm{B}^{\circ} / \mathrm{B}^{\circ}$, > 12 ans, pas de donneur de moelle osseuse HLA identique.

La réalité est que les prix avancés aujourd'hui sont définis avant tout par la maximisation du profit exigé par les actionnaires de l'industrie pharmaceutique. Ce secteur est en effet particulièrement performant à cet égard, ses marges bénéficiaires excédant celles de la plupart des autres secteurs du monde économique (données de la New York university Stern school of business) [17]. En fait, le retour sur investissement des grandes entreprises pharmaceutiques dépasse de $3 \%$ par an celui des autres secteurs économiques $[18,19]$. Ainsi, un euro investi en 1990 a rapporté en moyenne $44 €$ en 2015 pour l'ensemble des secteurs économiques mais $114 €$ pour le seul secteur pharmaceutique. Certes, la profitabilité est indispensable pour maintenir et stimuler l'innovation dans un secteur dont la recherche est le moteur. Mais lorsque les principes fondamentaux d'accès équitable aux soins de santé sont menacés, n'est-il pas raisonnable de limiter le profit de ce secteur au niveau de celui des autres secteurs tout aussi innovants? D'autant que dans le domaine qui nous occupe, on ne peut compter, en tous les cas à court terme, ni sur la compétition entre les firmes, ni sur l'introduction de médicaments génériques, pour entraîner la baisse des prix compte tenu de la technicité de production de ces médicaments (en particulier pour les thérapies cellulaires et géniques).

\section{Vers une innovation thérapeutique socialement responsable?}

Aujourd'hui, les organismes payeurs et les gouvernements tentent de contrôler la situation vaille que vaille en négociant, la plupart du temps dans le secret, des réductions de prix avec les industriels et en mettant en place des fonds de solidarité pour faire face aux situations les plus dramatiques. Cette opacité est justifiée par la possibilité d'obtention de rabais sur le prix facial affiché, mais observons que cette négociation n'est pas forcément à l'avantage de l'instance régulatrice, le Comité économique des produits de santé (CEPS) en France, qui n'a pas connaissance des prix de vente dans les autres pays, alors que l'industriel dispose de cette information, situation de négociation connue pour être désavantageuse [20-23]. Si ces deux mesures permettent encore de répondre partiellement aux attentes des patients, il est clair qu'elles seront insuffisantes pour assurer l'accès du plus grand nombre aux nouvelles avancées thérapeutiques qui se profilent. L'ICER estime que la mise au point d'une thérapie génique au prix par patient de 1 million de dollars US pour seulement $1 \%$ des patients atteints de maladies rares doublerait les dépenses en médicaments [24]. Dès aujourd'hui, se pose ainsi la question du prix du nouveau médicament utilisé pour le traitement de la mucoviscidose (Trikafta ${ }^{\circledR}$ ), qui devrait bénéficier à 6000 patients environ en France. Au prix affiché aujourd'hui aux États-Unis (de l'ordre de $300000 \$$ par an, soit environ 270000 euros par an), ce seul traitement mobiliserait un peu plus de $5 \%$ des dépenses annuelles de médicaments en France.

Si l'on veut éviter le bouleversement de nos systèmes de santé et préserver les intérêts de toutes les parties prenantes, l'heure est venue de revoir en profondeur les mécanismes qui gouvernent la fixation des prix. Cette remise en cause implique tant les industriels que les autorités publiques. En fait, les industries pharmaceutiques se préparent d'ores et déjà à des changements dont elles perçoivent bien qu'ils sont inéluctables. Ceuxlà s'inscrivent dans un contexte général où les entreprises et ceux qui les financent déclarent s'engager à privilégier 


\begin{tabular}{|c|c|c|c|c|}
\hline Maladie & Produit & $\begin{array}{l}\text { Année de } \\
\text { commercialisation }\end{array}$ & $\begin{array}{l}\text { Prix affiché } \\
\text { (€) }\end{array}$ & $\begin{array}{c}\text { Nombre estimé de patients éligibles } \\
\text { (Amérique du Nord + Europe) }\end{array}$ \\
\hline Amyotrophie spinale & Spinraza ${ }^{\circledR}$ & $\begin{array}{l}2016 \text { (USA) } \\
2017 \text { (Eur) }\end{array}$ & $\begin{array}{l}500000 € \text { puis } \\
250000 € / \text { an }\end{array}$ & $>1500$ \\
\hline Mucoviscidose & Trikafta $^{\circledR}$ & 2019 (USA) & $300000 \$ / a n$ & 60000 \\
\hline $\begin{array}{l}\text { Hémoglobinurie } \\
\text { paroxystique nocturne, } \\
\text { Syndrome hémolytique } \\
\text { et urémique }\end{array}$ & Soliris ${ }^{\circledR}$ & $\begin{array}{l}2007 \text { (Eur) } \\
2011 \text { (Eur) }\end{array}$ & $200000 € /$ an & $>4000$ \\
\hline Maladie de Gaucher & Cerezyme $^{\circledR *}$ & 2010 (Eur) & $70000 € /$ an & 2000 \\
\hline $\begin{array}{l}\text { Mucopolysaccharidose } \\
\text { de type IV }\end{array}$ & $\operatorname{Vimizim}^{\circledR}$ & 2015 (Eur) & $500000 € / a n$ & 100 \\
\hline $\begin{array}{l}\text { Maladie de Crohn, } \\
\text { rectocolite hémorragique }\end{array}$ & $\begin{array}{l}\text { Entyvio }{ }^{\circledR} \\
\text { anti-inté- } \\
\text { grine } \alpha 4 \beta 7\end{array}$ & 2015 (Eur) & $12000 € /$ an & $>200000$ \\
\hline $\begin{array}{l}\text { Mélanome métastatique, } \\
\text { cancer du poumon } \\
\text { non à petites cellules }\end{array}$ & Opdivo ${ }^{\circledR}$ & 2015 (Eur) & $60000 €$ & $>30000$ \\
\hline
\end{tabular}

Tableau II. Exemples de prix affichés de médicaments nouveaux onéreux. * : 2 molécules équivalentes sont commercialisées par d'autres laboratoires.

les investissements durables, affirmant leurs responsabilités en matière d'environnement, de mission sociétale et de gouvernance [25]. Restent pourtant à traduire ces belles intentions en actions concrètes. Dans le domaine pharmaceutique, il s'agit notamment de développer des modèles financiers et commerciaux garantissant des prix soutenables pour les organismes payeurs. Leur mise en œuvre nécessitera des modifications de gouvernance qui pourront s'inspirer de celles des « entreprises à mission sociétale » (benefit corporation) [26]. Ce statut, qui fait l'objet d'une certification externe indépendante, a déjà été adopté par une entreprise pharmaceutique et le sera bientôt par une grande multinationale française dont l'activité principale est centrée sur l'alimentaire. II implique que la mission légale de l'entreprise ne soit pas restreinte à l'intérêt des actionnaires, mais prenne en compte d'autres paramètres relatifs aux services rendus à la société en général. Dans la pratique, cela devrait conduire à inclure dans le conseil d'administration des représentants de la société civile et à revoir le mode de rémunération des dirigeants pour associer celui-ci à d'autres critères de performance que le seul profit ; on songe notamment au nombre et à la qualité des produits approuvés par les agences réglementaires ou mis sur le marché. Pour inciter les entreprises ou leurs filiales à modifier leur statut dans ce sens, nous proposons que les autorités publiques accordent des conditions de remboursement préférentielles aux médicaments qu'elles produisent.

La première étape dans le développement des médicaments est habituellement le transfert d'une découverte faite dans une institution publique ou académique vers une entreprise privée, souvent une startup ou un spin-off. Ce premier jalon va influencer toute la chaîne de valeurs qui conduit à la mise sur le marché du médicament issu de la découverte initiale. II est donc essentiel que les conditions de ce transfert garantissent autant que possible un prix final soutenable par les pouvoirs publics, et donc un retour équitable vers les citoyens dont les impôts ou les dons ont financé la recherche originale. Nous proposons donc d'inclure des clauses de prix raisonnable dans ces accords de transfert, des engagements qui devront rester applicables tout au long du développement jusqu'à la mise sur le marché du nouveau traitement. En parallèle, il est essentiel de préserver des conditions de commercialisation permettant aux industries un retour sur leur investissement qui soit décent et adapté aux normes du marché. II s'agit ici de fournir l'incitation nécessaire pour soutenir l'innovation dans des domaines spécifiques comme les maladies rares ou les pathologies particulièrement complexes.

Reste la question essentielle qui ne pourra être réglée qu'à l'échelon européen, voire au-delà : comment déterminer le prix juste et raisonnable d'un médicament, à la fois soutenable par les pouvoirs publics et suffisamment attractif pour les investisseurs et les actionnaires? II devra être fixé par une analyse multiparamétrique transparente considérant non seulement les investissements et les coûts de production consentis par l'industriel, mais aussi la valeur médicale ajoutée par rapport aux traitements préexistants et les investissements publics qui ont soutenu la recherche académique. Le temps est venu pour les différentes parties 
prenantes de s'engager sans préjugés dans un nouveau dialogue pour définir le modus operandi de cette analyse. Lorsqu'il s'avérera impossible de fixer un prix suffisant pour assurer la profitabilité du traitement (comme dans le cas de maladies très rares), une solution pourra être d'envisager la création d'entités de production de médicaments à but non lucratif comme celles créées pour les maladies des pays en voie de développement (DNDi - Drugs for neglected disease initiative) $[27,31](\rightarrow)$ ou de médicaments délaissés [28]. Un investissement public initial, ainsi que des contributions d'institutions philanthropiques et caritatives pourraient permettre le développe- $\rightarrow$ Voir l'éditorial de B.Pécoul, $m / s$ $n^{\circ} 12$, décembre 2016, page 1049 ment d'un petit nombre d'entités de ce type à l'échelle européenne. II s'agit maintenant de mobiliser l'intelligence collective - c'est-à-dire celle qui intègre les intérêts des différentes parties prenantes - pour dégager les solutions équilibrées qui feront bénéficier la société dans son ensemble des remarquables avancées de la science biomédicale. $\diamond$ What is the fair price of innovative therapy?

\section{LIENS D'INTÉRÊT}

Les auteurs déclarent n'avoir aucun lien d'intérêt concernant les données publiées dans cet article.

\section{RÉFÉRENCES}

1. High KA, Roncarolo MG. Gene Therapy. N Engl J Med 2019 ; 381 : 455-64.

2. Fischer A, Hacein-Bey-Abina S. Gene therapy for severe combined immunodeficiencies and beyond. J Exp Med 2020 ; 217 : e20190607.

3. Editorial. Gene therapy's next installment. Nat Biotechnol $2019 ; 37: 697$.

4. Bach PB. National coverage analysis of CAR-T therapies - Policy, evidence, and payment. $N$ Engl J Med 2018 ; 379 : 1396-8.

5. Mazzucato M. High cost of new drugs. BMJ $2016 ; 354: i 4136$.

6. Fischer A, Dewatripont M, Goldman M. Benefit corporation: a path to affordable gene therapies? Nat Med $2019 ; 25: 1813-4$.

7. Middleton PG, Mall MA, Drevinek P, et al. Elexacaftor-tezacaftor-ivacaftor for cystic fibrosis with a single Phe508del allele. N Engl J Med 2019 ; 381 : 1809-19.

8. Collins FS. Realizing the dream of molecularly targeted therapies for cystic fibrosis. $N$ EnglJ Med 2019 ; $381: 1863-5$.

9. Bach PB. Anchoring' was at work in setting the price of Novartis' new gene therapy. https:// www.statnews.com/2019/2006/2004/anchoring-price-zolgensma/. June 4, 2019.

10. Evaluate Pharma. World Preview 2019, Outlook to 2024 (2019). https://www.evaluate.com/ thought-leadership/pharma/evaluatepharma-world-preview-2019-outlook-2024

11. Stevens AJ, Jensen JJ, Wyller K, et al. The role of public-sector research in the discovery of drugs and vaccines. $N$ Engl J Med $2011 ; 364: 535-41$.

12. Nayak RK, Avorn J, Kesselheim AS. Public sector financial support for late stage discovery of new drugs in the United States: cohort study. BMJ 2019 ; 367: 15766.
13. Sarpatwari A, LaPidus AK, Kesselheim AS. Revisiting the National institutes of health fair pricing condition: promoting the affordability of drugs developed with government support. Ann Intern Med 2020.

14. World Health Organization. Improving the transparency of markets for medicines, vaccines, and other health products. SEVENTY-SECOND WORLD HEALTH ASSEMBLY72/A/CONF./2 Rev.1 Agenda item 11.7; 28 May 2019: https://apps.who.int/gb/ebwha/pdf_files/WHA72/A72_ACONF2Revl-en.pdf

15. Luzzatto L, Hyry HI, Schieppati A, et al. Outrageous prices of orphan drugs: a call for collaboration. Lancet $2018 ; 392: 791-4$.

16. Green A. Biotech companies defend prices of one-off gene therapy. Financial Times https://www.ft.com/content/edd639fc-9755-1le998b9-e38c177b152f: December 9, 2019

17. New York University Stern school of business. httpi//bit.ly/2IIHwZw

18. Thakor RT, Anaya N, Zhang Y, et al. Just how good an investment is the biopharmaceutical sector? Nat Biotechnol 2017 ; 35 : 1149-57.

19. Tay-Teo K, Ilbawi A, Hill SR. Comparison of sales income and research and development costs for FDA-approved cancer drugs sold by originator drug companies. JAMA Netw Open 2019; 2 : el86875.

20. Bolton P, Dewatripont M. Contract theory. Cambridge : The MIT Press, 2004.

21. Hart 0. Firms, contracts, and financial structure. Clarendon Press-0xford University Press, 1995.

22. Wineinger NE, Zhang, Topol $\varepsilon$ J. Trends in prices of popular brand-name prescription drugs in the United States. JAMA Netw Open 2019; 2 : el94791.

23. La Cour des Comptes. Le rapport public annuel 2017. https://www. ccomptes.fr/fr/publications/le-rapport-public-annuel-2017. 8 février 2017.

24. Marsden G, Towse A, Pearson SD, et al. Gene therapy: understanding the science, assessing the evidence, and paying for value. ICER - Institute for clinical and economic review, a report from the 2016 ICER membership policy. March 2017.

25. Business rountable. Our commitment. https://opportunitybusinessrountable.org/ ourcommitment. Released: 2019 ; signatures updated: September 2019, December 2019, February 2020.

26. Cummings $B$. Benefit corporations : how to enforce a mandate to promote the public interest. Columbia Law Rev 2012 ; 112 : 578-627.

27. DNDi - Drugs for Neglected Disease Initiative. https://dndi.org

28. Nielsen TB, Brass EP, Gilbert DN, et al. Sustainable discovery and development of antibiotics - Is a nnonprofit approach the future? $N$ Engl J Med $2019 ; 381: 503-5$.

29. Barkats M. SMA: de la découverte du gène à la thérapie génique. Med Sci (Paris) $2020 ; 36: 137-40$.

30. Braun $\mathrm{S}$. Thérapies génétiques de l'amyotrophie spinale infantile : un morceau d'histoire de la médecine. Med Sci (Paris) 2020 ; 36 : 141-6.

31. Pécoul B. Un modèle alternatif et innovant de recherche et développement pour garantir l'accès aux médicaments. Maladies négligées, populations négligées et système d'innovation biomédicale. Med Sci (Paris) 2016 ; 32 : 1049-50.

TIRÉS À PART

A. Fischer

\section{LA FONDATION PREMUP : UN OPÉRATEUR DE TERRAIN EN PÉRINATALITÉ RECONNU POUR SON EXCELLENCE ET SON INTERDISCIPLINARITÉ}

La Fondation de coopération scientifique PremUp, unique en Europe, intervient sur la prévention du handicap à la naissance, par la protection de la santé de la femme enceinte et du nouveau-né.

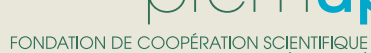
SUR LA GROSSESSE ET LA PRÉMATURITÉ

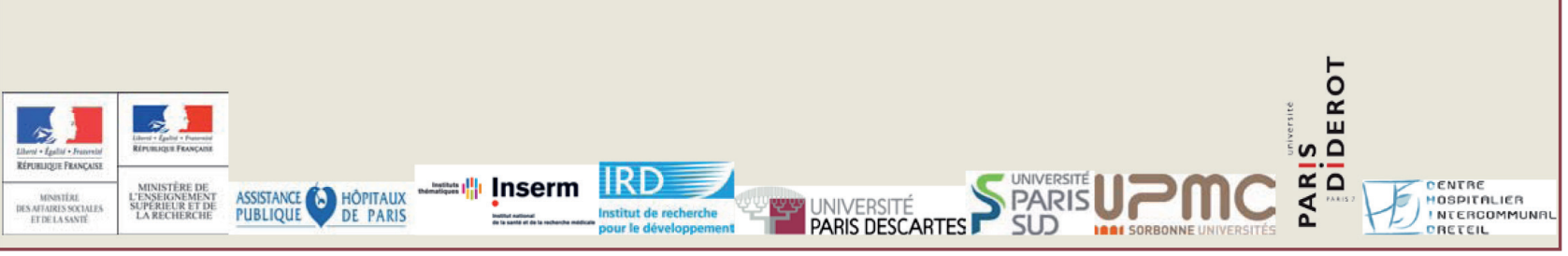

